\title{
CAMSAP1 Mutation Correlates With Improved Prognosis in Small Cell Lung Cancer Patients Treated With Platinum-Based Chemotherapy
}

OPEN ACCESS

Edited by:

Lianbo Li,

University of Texas Southwestern Medical Center, United States

Reviewed by:

Qing Hu,

University of Texas Southwestern Medical Center, United States

Yaru Xu,

University of Texas Southwestern Medical Center, United States Xinyi Zhang,

University of Texas Southwestern Medical Center, United States

*Correspondence:

Weitao Shen

shenweitao1@sina.com

Jian Zhang

zhangjian@i.smu.edu.cn

Peng Luo

luopeng@smu.edu.cn

${ }^{+}$These authors have contributed equally to this work and share first authorship

Specialty section:

This article was submitted to Molecular and Cellular Oncology, a section of the journal Frontiers in Cell and Developmental Biology

Received: 05 September 2021 Accepted: 16 December 2021 Published: 11 January 2022

Citation:

Yi Y, Qiu Z, Yao Z, Lin A, Qin Y, Sha R, Wei $T$, Wang $Y$, Cheng $Q$, Zhang J, Luo $P$ and Shen W (2022) CAMSAP1 Mutation Correlates With Improved Prognosis in Small Cell Lung Cancer

Patients Treated With PlatinumBased Chemotherapy. Front. Cell Dev. Biol. 9:770811. doi: 10.3389/fcell.2021.770811

\author{
Yonglin $\mathrm{Yi}^{1+}$, Zhengang Qiu ${ }^{2 \dagger}$, Zifu Yao ${ }^{1,3+}$, Anqi Lin $^{1+}$, Yimin Qin ${ }^{1}$, Ruizhan Sha ${ }^{1}$, Ting Wei ${ }^{1}$, \\ Yanru Wang ${ }^{1}$, Quan Cheng ${ }^{4}$, Jian Zhang ${ }^{1 *}$, Peng Luo ${ }^{1 *}$ and Weitao Shen ${ }^{1 *}$ \\ ${ }^{1}$ Department of Oncology, Zhujiang Hospital, Southern Medical University, Guangzhou, China, ${ }^{2}$ Department of Oncology, First \\ Affiliated Hospital of Gannan Medical University, Guangzhou, China, ${ }^{3}$ The First Clinical Medical School, Southern Medical \\ University, Guangzhou, China, ${ }^{4}$ Department of Neurosurgery, Xiangya Hospital, Center South University, Changsha, China
}

Platinum-based chemotherapy is the first-line treatment for small cell lung cancer (SCLC). However, due to patients developing a resistance to the drug, most experience relapse and their cancer can become untreatable. A large number of recent studies have found that platinum drug sensitivity of various cancers is affected by specific gene mutations, and so with this study, we attempted to find an effective genetic biomarker in SCLC patients that indicates their sensitivity to platinum-based drugs. To do this, we first analyzed whole exome sequencing (WES) and clinical data from two cohorts to find gene mutations related to the prognosis and to the platinum drug sensitivity of SCLC patients. The cohorts used were the Zhujiang cohort $(N=138)$ and the cohort reported by George et al. $(N=101)$. We then carried out gene set variation analysis (GSVA) and gene set enrichment analysis (GSEA) to investigate possible molecular mechanisms through which these gene mutations affect patient prognosis and platinum drug sensitivity. We found that for SCLC patients, CAMSAP1 mutation can activate anti-tumor immunity, mediate tumor cell apoptosis, inhibit epithelial-mesenchymal transition (EMT), improve prognosis, and improve platinum drug sensitivity, suggesting that CAMSAP1 mutation may be a potential biomarker indicating platinum drug sensitivity and patient prognosis in SCLC.

Keywords: small cell lung cancer, CAMSAP1, platinum-based chemotherapy, drug sensitivity, biomarker

\section{INTRODUCTION}

Lung cancer is one of the most common malignant tumors in the world, and it is divided into two categories: small cell lung cancer and non-small cell lung cancer. Small cell lung cancer (SCLC) accounts for $13-15 \%$ of all lung cancers and has a high degree of malignancy, having a 5 -year survival rate of less than 7\% (Govindan et al., 2006; Sabari et al., 2017; Farago and Keane, 2018; Tsoukalas et al., 2018; Qiu et al., 2019a; Li et al., 2020). In 70\% of SCLC patients, extended-stage SCLC (ES-SCLC) was diagnosed. Platinum-based chemotherapy is the first-line treatment of ES-SCLC with an effective rate

\footnotetext{
Abbreviations: SCLC, small cell lung cancer; ES-SCLC, extended-stage SCLC; WES, whole exome sequencing; GSVA, gene set variation analysis; GSEA, gene set enrichment analysis; cMAP; Connectivity Map; EMT, epithelial-mesenchymal transition; MoA, mode-of-action; EP, Etoposide combined with cisplatin; IP, irinotecan combined with cisplatin; ORR, overall response rate; OS, overall survival; PFS, progression-free survival.
} 


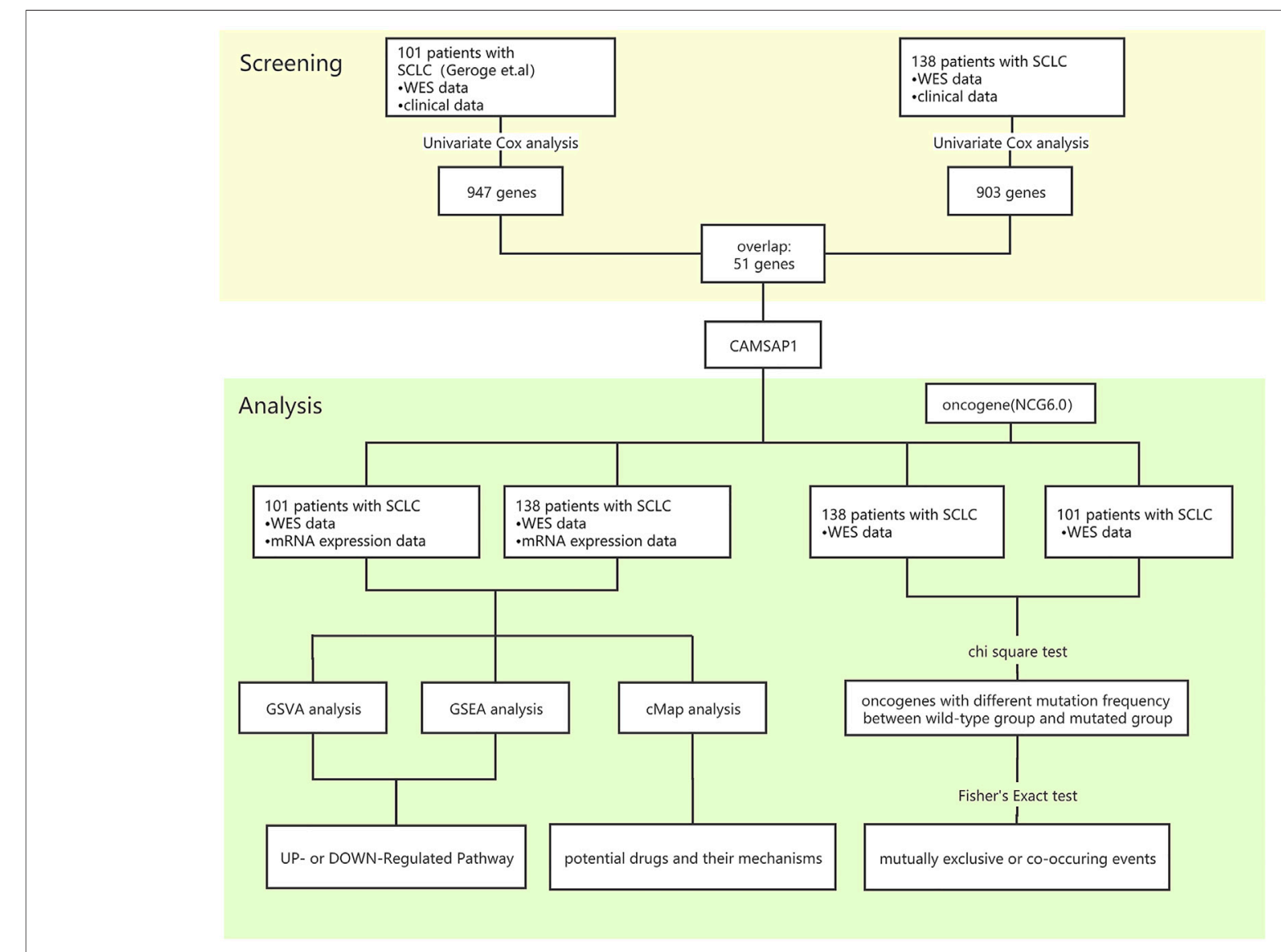

FIGURE 1 | Bioinformatics analysis process. SCLC, small-cell lung cancer; GSVA, gene set variation analysis; GSEA, gene set enrichment analysis; cMAP, connectivity map.

of $50-75 \%$ in the initial stage of treatment (Rossi et al., 2012; Waqar and Morgensztern, 2017). However, most patients relapse within 6 months due to the development of drug resistance, leading to disease progression and in some cases, death (Sabari et al., 2017). Topotecan is the only second-line drug certified by the FDA for the treatment of ES-SCLC, and is mainly effective in the patients who are also sensitive to first-line treatment (Ardizzoni et al., 1997).

The development of chemotherapy resistance occurs through many mechanisms, such as a decrease in drug accumulation and apoptosis, an increase in drug inactivation, cell protective autophagy, and the number of cancer stem cells (Hermann et al., 2007; Mani et al., 2015; Luo et al., 2017; Maji et al., 2018; Wang et al., 2018; Qiu et al., 2019b). The number of cancer stem cells is closely related to epithelial-mesenchymal transition (EMT) and the immune microenvironment (Ahmed et al., 2018; Li et al., 2019). Additionally, the activation of antitumor immune activity has been shown to reverse chemotherapy resistance by increasing the rate of apoptosis in tumor cells (Xu et al., 2016). Therefore, to improve the prognosis of patients with SCLC, it is very important to find effective biomarkers indicative of platinum drug sensitivity and to explore the mechanisms that affect platinum drug sensitivity.
In recent years, a large number of studies have found that gene mutations can affect the platinum drug sensitivity of various cancers. For example, it has been shown that the overexpression of CCDC69 can activate the p14 ARF/MDM2/p53 pathway in ovarian cancer and that it is associated with a higher sensitivity to platinum drugs (Cui et al., 2019). Also, Qiang Li found that ERCC2 mutation can abrogate nuclear error repair in bladder cancer, thus increasing platinum drug sensitivity ( $\mathrm{Li} \mathrm{Q}$. et al., 2019). In one more example, I. Lohse was able to show that BRCA1, BRCA2 mutations can improve platinum drug sensitivity of pancreatic cancer via accumulation of DNA damage (Lohse et al., 2015). Despite this wealth of research, the relationship between gene mutations and sensitivity to platinum drugs in SCLC is not clear. Therefore, with this study we explored the mutant genes from two SCLC cohorts in order to determine whether they could be used as biomarkers that indicate platinum drug sensitivity and patient prognosis.

Calmodulin-regulated spectrin-associated protein 1 (CAMSAP1), is a member of CAMSAP/Patronin/Nezha family (Hendershott and Vale, 2014). This family localizes on the microtubule minus-end, promoting microtubule stability (Chuang et al., 2014; Hendershott and Vale, 2014; Yau et al., 

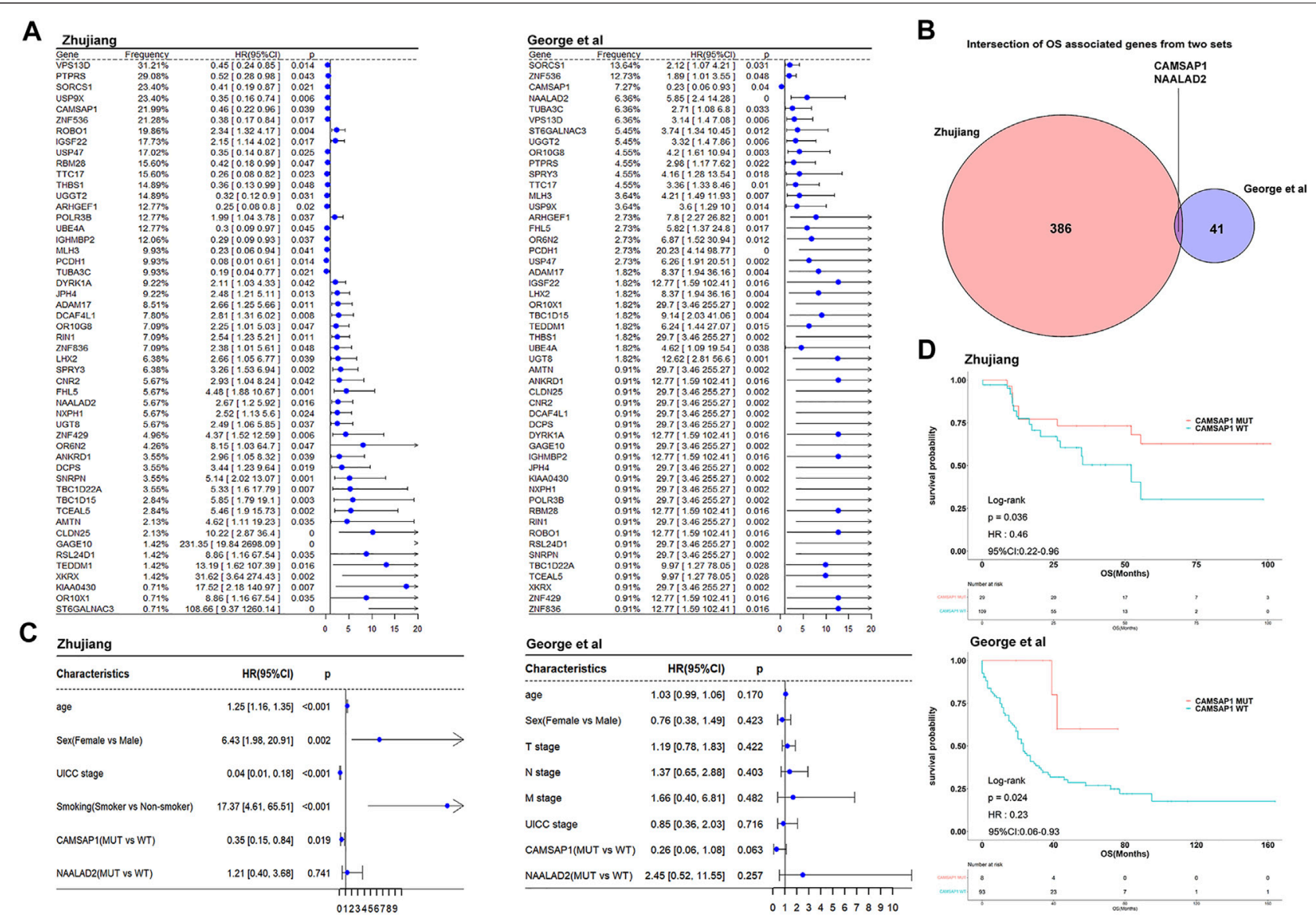

FIGURE 2 | Cox proportional risk regression analysis identifying survival-related genes. (A) The forest plots show the univariate cox analysis results of 51 common OS-related gene mutations in the Zhujiang and reported cohorts (B) Venn diagram showing the overlap of OS-related genes with mutation frequency $>5 \%$ in the Zhujiang and reported cohorts. (C) Multivariate cox analysis results of gene mutations, clinical factors and OS in the Zhujiang and reported cohorts. (D) Kaplan-Meier survival analysis results of OS comparing the CAMSAP1-MT group (red) and the CAMSAP1-WT group (blue) in the Zhujiang and reported cohorts. OS, overall survival; HR, hazard ratio; CL, confidence interval.

2014; Gong et al., 2018). The CKK domain (DUF1781) is in the C-terminal of proteins of CAMSAP family, which binds microtubules (Baines et al., 2009) and mediates the association of CAMSAPs with microtubule minus-end (Gong et al., 2018). The $\mathrm{CH}$ domain is in the N-terminus of CAMSAPs and is involved in the regulation of actin dynamics CAMSAPs (Baines et al., 2009; Gong et al., 2018). CAMSAP1 is associated with better prognosis in acute lymphoblastic leukemia (Wang et al., 2015). However, there is no report about the role of CAMSAP1 in SCLC.

In this study, whole exome sequencing (WES) data and clinical information from a reported SCLC cohort (reported by George et al., (2015) and an SCLC cohort from Zhujiang Hospital and Sun Yat-sen University Cancer Center, were used to explore the relationships between specific gene mutations, improved prognosis, and increased platinum-based chemotherapy sensitivity. In addition, we also attempted to identify the related mechanisms and potential drugs. Our results show that
CAMSAP1 mutation can be used as a prognostic marker in SCLC patients undergoing treatment with platinum-based chemotherapy. CAMSAP1 mutation was associated with better overall survival (OS), and it shows the potential to increase platinum drug sensitivity in patients with SCLC. Analyses of the mechanism showed that CAMSAP1 mutation can activate anti-tumor immunity, mediate apoptosis of tumor cells and inhibit EMT. This study proves that CAMSAP1 mutation can play an important role in predicting prognosis and platinumbased chemotherapy sensitivity in patients with SCLC.

\section{METHODS}

\section{Clinical Cohort and Expression Data}

To assess the relationship between gene mutations and platinum drug sensitivity of SCLC, we collected clinical and WES data from two cohorts. The Zhujiang cohort included 138 


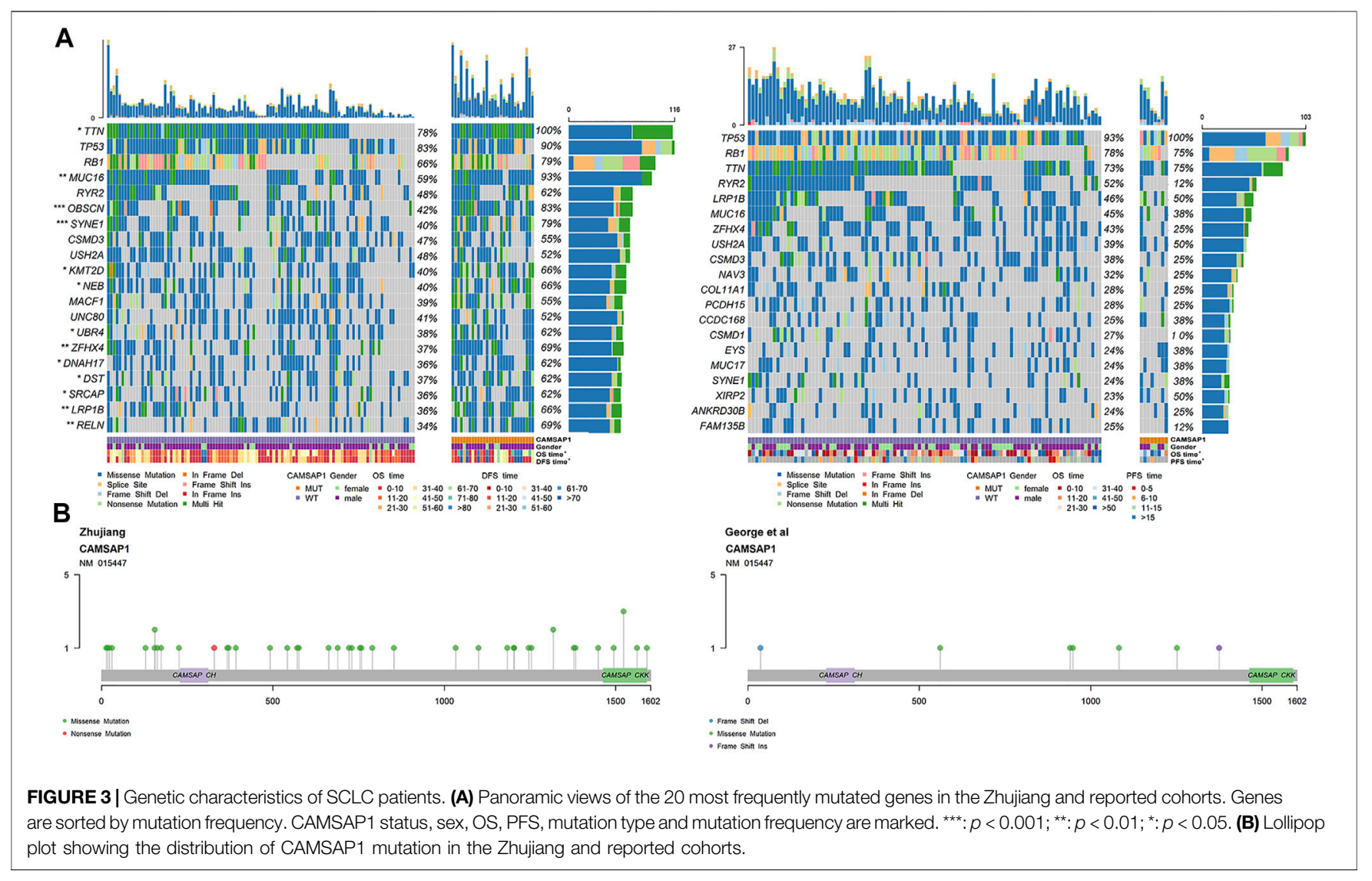

SCLC patients who were treated with platinum-based chemotherapy in Zhujiang hospital and Sun Yat-Sen University Cancer Center. The reported cohort was downloaded from George's study and included 101 SCLC patients who were treated with platinum-based chemotherapy. In addition, we obtained the gene expression file for patients in the Zhujiang cohort.

\section{Identification of Survival-Related Genes}

Genes needed to meet the following conditions to be considered survival-related genes in SCLC: 1) the relationship identified by univariate Cox proportional hazards analysis between gene mutation and OS was statistically significant $(p<0.05) ; 2)$ the frequency of the gene mutation was $>5 \%$, and; 3 ) the common genes were identified in both the Zhujiang cohort and the reported cohort (Figure 1).

\section{The Mutation Status of Oncogenes and the Relationship Between Gene Mutations}

The NCG6.0 database (http://ncg.kcl.ac.uk/) consists of protein, expression, and functional data for 2,372 cancer genes. We used this database to assist in screening out oncogene mutations from the two cohorts. The $\mathrm{R}$ package "maftools" was used to identify mutual exclusivity and cooccurrence among gene mutations.

\section{Enrichment Analysis of Differentially Expressed Genes and Pathways}

The R package "limma" was used to identify differentially expressed genes (DEGs) in the CAMSAP1-MT group and CAMSAP1-WT group. The calculation formula of the LogFC cut-off value is:

$$
|\overline{\log F C}|+2^{\star} \sigma(|\log F C|)
$$

The LogFC cut-off value was 0.766 in the Zhujiang cohort and 0.723 in the reported cohort. We used heatmaps to show the expression of the top 20 DEGs with the largest fold change (FC) between the CAMSAP1-MT and CAMSAP1-WT groups, and volcano plots to visualize the DEGs. Using the C2 collection (curated gene sets) obtained from the Molecular Signatures Database of the Broad Institute (MSigDB), we calculated the GSVA score for each patient using the R package "GSVA". Patients were divided into high- and low-GSVA score groups according to the median GSVA score, and a Kaplan-Meier survival analysis was performed on the two groups. Enrichment analysis of gene annotation (GSEA) was performed by the $\mathrm{R}$ package "ClusterProfiler". When comparing pathway differences in the $\mathrm{C} 2$ collection (curated gene sets), $p<0.05$ was considered to be significant. Then we selected the cancer-associated pathways using PubMed dataset (https://pubmed.ncbi.nlm.nih.gov/). 


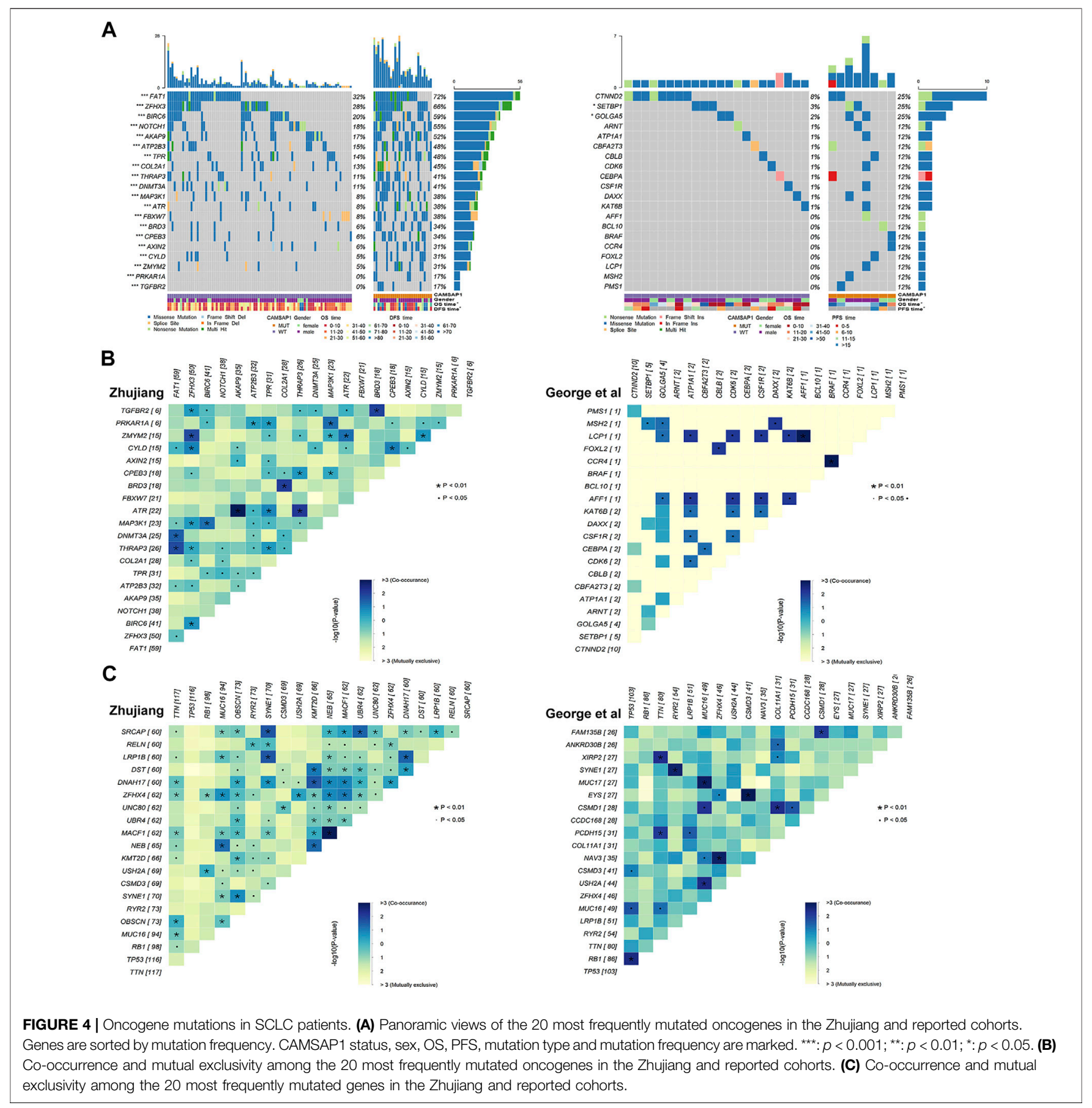

\section{Identification of Potential Drugs}

In order to identify potential small molecule drugs which target CAMSAP1, we used Connectivity Map (cMAP) database, which is a library of expression files of cell lines treated with different small molecule drugs. We divided the DEGs of the two cohorts into up- and down-regulation groups and entered them into the cMAP online tool (https://portals.broadinstitute.org/cmap/) to obtain a permutation result. cMAP tools (https://clue.io/) were then used to explore the mode-of-action (MoA).

\section{Statistical Analysis}

The relationship between gene mutation frequency and CAMSAP1 status was determined by chi-square test. The relationships between gender, GSVA score and CAMSAP1 


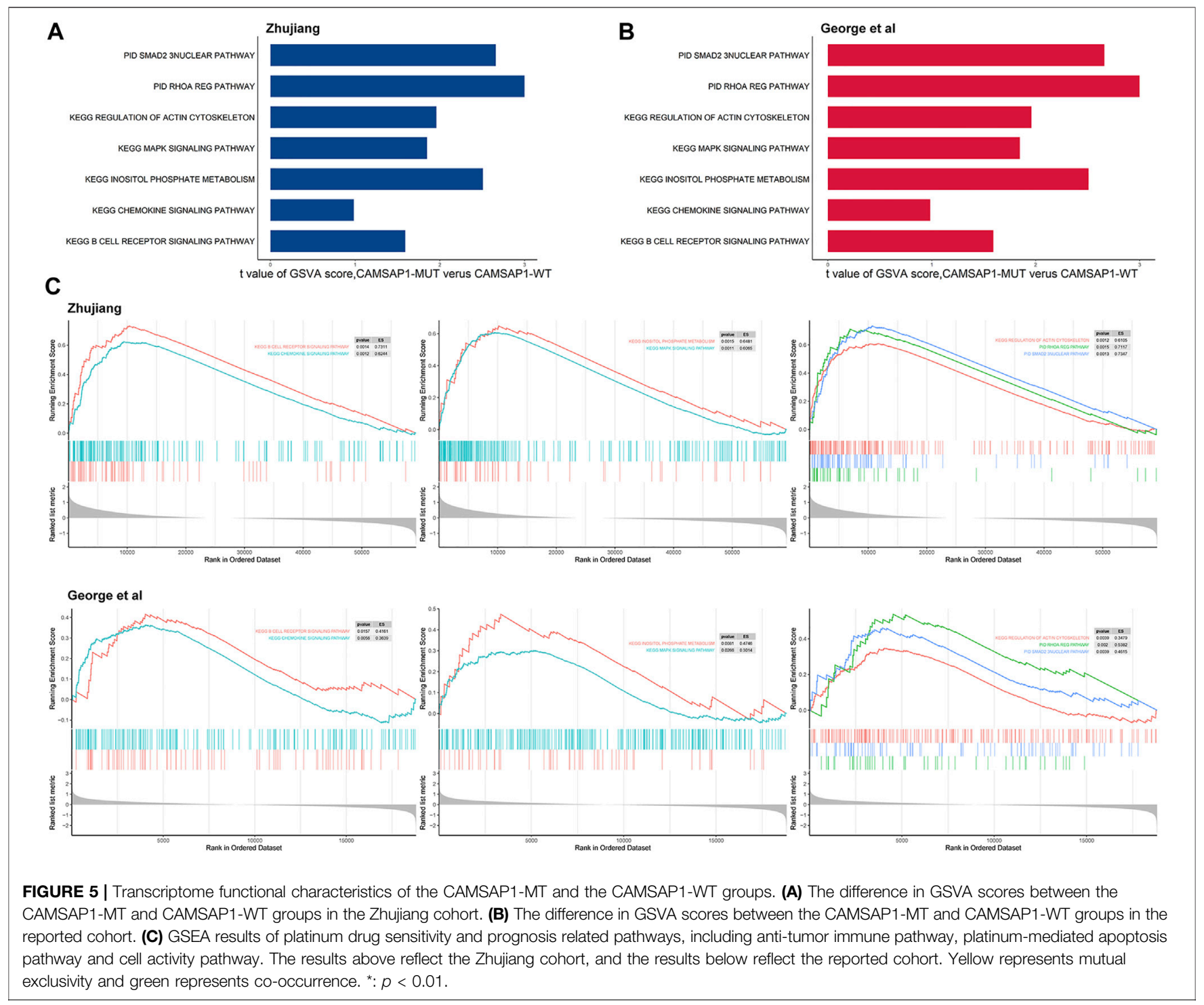

status were also determined by chi-square test. To determine the relationship between enrichment score and CAMSAP1 status, permutation test was used, and for the relationships between OS, progression-free survival (PFS) and CAMSAP1 status log-rank test was used. The R package "survival" and "survminer" were utilized to conduct Kaplan-Meier survival analyses, and the relationships between OS, PFS, clinical characteristics and gene mutations were determined by univariate/multivariate cox proportional hazards analysis. Co-occurrence or mutual exclusivity events among gene mutations were identified by Fisher's Exact test. For all tests, $p<0.05$ was considered to be statistically significant. All statistical analysis was conducted by $\mathrm{R}$ software (version 4.1 ), and the R package "maftools" was used to analyze and visualize the MAF files.

\section{RESULTS}

\section{CAMSAP1 Is a Prognostic Marker for SCLC Patients Receiving Platinum-Based Chemotherapy}

We used clinical and WES data from two cohorts of SCLC patients receiving platinum-based chemotherapy treatment. There were 138 patients in the Zhujiang cohort and 101 patients in the reported cohort. For both of these cohorts, no statistical difference in clinical characteristics between CAMSAP1-MT and CAMSAP1-WT groups was found (Supplementary Tables S1, S2). We used univariate cox analysis to explore the effect of gene mutations on OS and there were 51 common gene mutations of statistical 
significance in two cohorts $(p<0.05$, Figure 2A). After application of the condition that gene mutation frequency must be more than 5\%, there were 386 and 41 OS-related genes in the Zhujiang and reported cohorts respectively. CAMSAP1 and NAALAD2 were shared in both cohorts (Figure 2B). The mutation frequency of CAMSAP1 is $21.99 \%$ in Zhujiang cohort and $7.27 \%$ in the reported cohort. The results of our analysis showed that CAMSAP1 mutation was associated with better prognosis and NAALAD2 mutation was associated with worse prognosis. The univariate cox statistical index $(p<0.05)$ was introduced into multivariate cox. The results showed that in the Zhujiang cohort age, sex, UICC stage, smoking and CAMSAP1 mutation were independent factors affecting prognosis, and that no significant relationship between NAALAD2 and OS exists $(\mathrm{HR}=1.21,95 \% \mathrm{Cl} 0.40-3.68, p=$ $0.741)$. In the reported cohort, there was no independent factor affecting prognosis found (Figure 2C). Finally, we selected CAMSAP1 as a candidate molecule for a mutation related to increased drug sensitivity and better prognosis in both cohorts. To further explore the predictive function of CAMSAP1, we performed a Kaplan-Meier survival analysis that showed that patients in the CAMSAP1-MT group had a longer OS than those in the CAMSAP1-WT group in both the Zhujiang cohort $(\mathrm{HR}=0.46,95 \% \mathrm{Cl} 0.22-0.96, p=0.036)$ and the reported cohort $(\mathrm{HR}=0.23,95 \% \mathrm{Cl} 0.06-0.93, p=0.024)$ (Figure 2D). The relationship among CAMSAP1 mutation, CAMSAP1 expression and $O S$ had no statistical significance (Supplementary Figure S1).

\section{Relationship Between CAMSAP1 Status, Other Gene Mutations, and Clinical Characteristics}

Among the genetic landscape of 137 samples from the Zhujiang cohort and 108 samples from the reported cohort (Figure 3A), TP53, RB1, TTN, RYR2, MUC16, SYNE1, CSMD3, USH2A, ZFHX4 and LRP1B were the top 20 most frequently mutated genes. In the Zhujiang cohort, the mutation frequency of the following genes was higher in the CAMSAP1-MT group than in the CAMSAP1-WT group; TTN (100 vs. $78 \%$ ), MUC16 (93 vs. 59\%), SYNE1 (79 vs. $40 \%$ ), ZFHX4 (69 vs. $37 \%$ ), LRP1B (66 vs. $36 \%$ ). Missense mutation was the main mutation type of the following: TP53, TTN, RYR2, MUC16, SYNE1, CSMD3, USH2A, ZFHX4 and LRP1B. The RB1 mutations were predominantly splice site and nonsense mutations. In addition, we compared the clinical features (including gender, PFS and OS) of the CAMSAP1-MT group and CAMSAP1-WT group. In Zhujiang cohort, patients in the CAMSAP1-MT group showed a longer OS and PFS than those in the CAMSAP1-WT group. In the reported cohort, only OS in the CAMSAP1-MT group was longer. In both Zhujiang and reported cohorts, there was no significant difference in gender between the two groups. Figure 3B shows the main mutation sites of CAMSAP1 in both cohorts. The main mutation type can be seen here as missense mutation and is located at CAMSAP CKK and CAMSAP $\mathrm{CH}$ domain.

\section{The Interaction Among Mutant Genes and the Relationship Between CAMSAP1 Status and Oncogene Status}

In order to investigate the relationship between CAMSAP1 mutation and oncogene mutations in the two cohorts, we used WES and clinical data in combination with the NCG database to compare the oncogene characteristics in the CAMSAP1-MT and CAMSAP1-WT groups. In the landscape of the Zhujiang cohort (Figure 4A), the mutation frequency of oncogenes in the CAMSAP1-MT group was significantly higher than that in the CAMSAP1-WT group $(p<0.05)$. In the CAMSAP1-MT group, the oncogenes with a mutation frequency $>50 \%$ were FAT1 (72 vs. $32 \%$ ), ZHFX3 (66 vs. $28 \%$ ), BIRC 6 (59 vs. $20 \%$ ), NOTCH1 (55 vs. $18 \%$ ) and AKAP6 (52 vs. $17 \%$ ). The most common mutation type among these genes was missense mutation. In the reported cohort (Figure 4A), the oncogenes with the highest mutation frequency were CTNND2, SETBP1 and GOLGA5. For the reported cohort, the mutation frequency in the CAMSAP1-MT group was significantly higher than that in the CAMSAP1-WT group for SETBP1 (25 vs. 3\%) and GOLGA5 (25 vs. $2 \%$ ). The mutation frequency of other oncogenes was not significantly different between the two groups. In the Zhujiang cohort, ZHFX3 mutation was concurrent with BIRC6 mutation $(p<0.01)$ but mutually exclusive with FAT1 $(p<0.05)$. In the reported cohort, all oncogenes with statistical significance were concurrent (Figure 4B).

We also performed a co-occurrence and mutual exclusivity analysis among all gene mutations (Figure 4C). Among the common gene mutations in both the Zhujiang and reported cohorts, SYNE1 and RYR2, USH2A and MUC16, and MUC16 and TTN were concurrent in the Zhujiang cohort but mutually exclusive in the reported cohort.

\section{Transcriptome Characteristics Related to CAMSAP1 Mutation}

In both cohorts, we compared the expression data between the CAMSAP1-MT and CAMSAP1-WT groups by using the $\mathrm{R}$ package "limma" to identify the DEGs. In the Zhujiang cohort, compared with the expression in CAMSAP1-WT group, there were 830 up-regulated genes and 496 down-regulated genes in the CAMSAP1-MT group. In the reported cohort, compared with the expression in CAMSAP1-WT group, there were 250 up-regulated genes and 175 down-regulated genes in the CAMSAP1-MT group (Supplementary Figure S2A). The expression of top 20 genes with the largest LogFC in the CAMSAP1-MT group and CAMSAP1WT group is shown in Supplementary Figure S2B.

In order to determine the mechanism underlying the improved prognosis in the CASAP1-MUT group, GSVA and Kaplan-Meier analyses were used. In the CAMSAP1-MT group, GSVA scores of anti-tumor immunity, platinum-mediated apoptosis, and cell activity pathways were significantly higher than those in the CAMSAP1-WT group, and patients with high GSVA scores showed better prognosis than those with low GSVA scores (Figures 5A,B). For verification, we used the previously identified DEGs to carry out GSEA analysis. The results were consistent, also showing that anti-tumor immunity, platinum-mediated apoptosis 


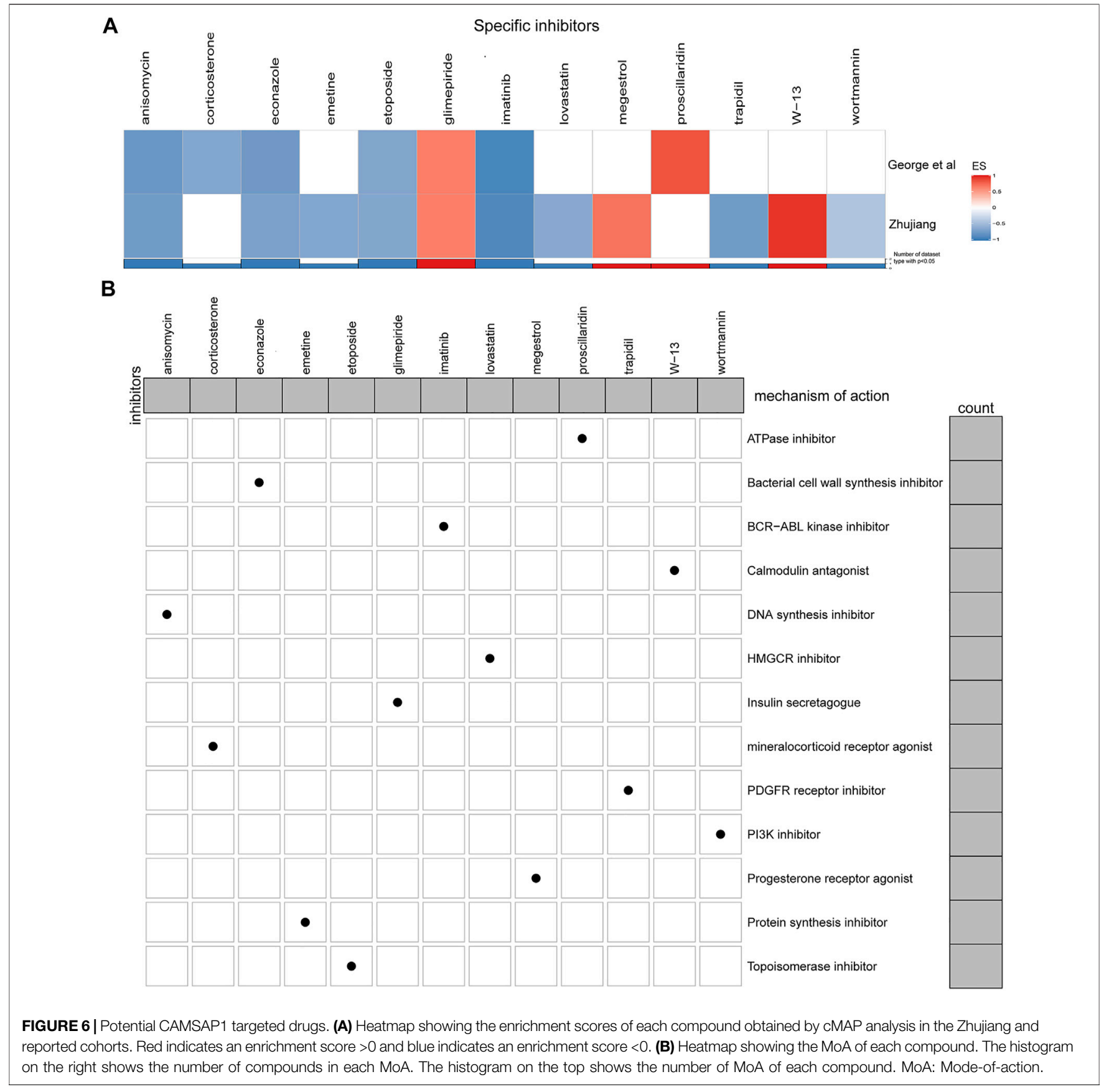

and cell activity pathways were significantly up-regulated in CAMSAP1-MT groups in the two cohorts (Figure 5C).

\section{Potential Therapeutic Drugs and Mode of Action Based on CAMSAP1 Mutation}

We utilized the cMAP database, inputting up- and downregulated genes, and searched for potential drugs that target
CAMSAP1. Eleven of the known tumor therapeutic drugs were enriched in the Zhujiang cohort, and seven in the reported cohort $(p<0.05)$. There were five drugs that were enriched in both cohorts, and thus, identified as being potential therapeutic drugs for SCLC patients with CAMSAP1 mutation. These were: anisomycin, econazole, etoposide, glimepiride and imatinib (Figure 6A). MoA is a group of drugs with similar gene markers and therapeutic effects (Ma et al., 2013). Through cMap 


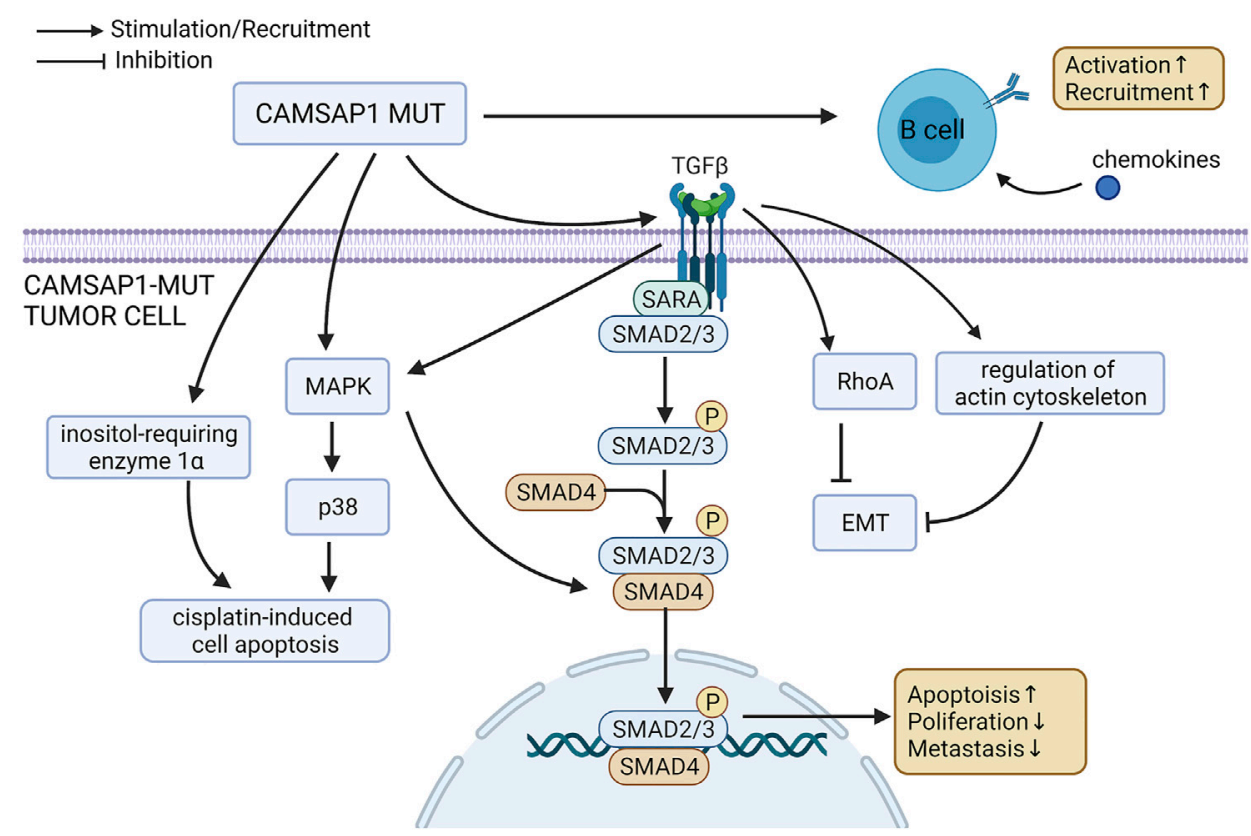

FIGURE 7 | Possible mechanism underlying the improved platinum drug sensitivity and prognosis of CAMSAP1-MT SCLC patients.

MoA analysis, we found that 13 drugs were enriched in 13 modes of action (Figure 6B).

\section{DISCUSSION}

Etoposide combined with cisplatin (EP) or irinotecan combined with cisplatin (IP) are the first-line treatments for SCLC patients (Ogino et al., 2016), and the overall response rate (ORR) is $57 \%$ (Rossi et al., 2012). However, most patients will develop resistance to these chemotherapy drugs and eventually die of recurrent diseases (Jett et al., 2013). Therefore, it is of great importance to find predictive markers which can be used to predict and improve platinum drug sensitivity in order to improve the prognosis of SCLC patients. Previous studies have shown that specific gene mutations are associated with higher platinum drug sensitivity in many cancers, such as CCDC69 mutation in ovarian cancer, ERCC2 mutation in bladder cancer, and BRCA mutation in pancreatic cancer (Lohse et al., 2015; Cui et al., 2019; Li Q. et al., 2019). Therefore, in this study we attempted to discover the gene markers related to the increased sensitivity of platinum drugs in patients with SCLC. We analyzed WES and clinical data from a reported SCLC cohort (reported by George et al. (2015) and an SCLC cohort from Zhujiang Hospital and Sun Yat-sen University Cancer Center. We found a significant relationship between CAMSAP1 mutation and both increased sensitivity to platinum drugs and the improved prognosis of SCLC patients treated with platinum-based chemotherapy.

Our analysis of oncogenes showed that the mutation frequency of some such as ZHFX3, BIRC6, Axin2, CPEB3, TPR, Axin2, Notch1, DNMT3A and COL2A1 was significantly higher in the CAMSAP1MT group compared to the CAMSAP1-WT group, and that this was closely related to improved prognosis. ZHFX3, BIRC6, Axin2, CPEB3 and TPR can regulate the proliferation and apoptosis of tumor cells. After silencing CPEB3, the growth of tumor cells increases while apoptosis decreases (Lin et al., 2019). Thus, CPEB3 mutation may inhibit the growth of tumor cells by increasing CPEB3 expression. ZHFX3 and BIRC6 can promote the proliferation of tumor cells, meaning that the mutation or knockout of these can inhibit the progression of cancer, resulting in a prolonged OS (Hao et al., 2020; Zhang et al., 2021). Of note, BIRC6 and ZHFX3 mutations always appear together (Figure 4). Silencing of TPR can trigger G0-G1 block, mediate cell aging through p53 and promote apoptosis of tumor cells (DavidWatine, 2011). Axin2 is a negative regulator of the $\mathrm{Wnt} / \beta$ catenin pathway and, once activated, can inhibit the proliferation and formation of tumors by inhibiting $\mathrm{Wnt} / \beta$-catenin (Yu et al., 2019). In addition, some Axin 2 mutant genotypes are closely related to the low-risk lung cancer types (Kanzaki et al., 2006; Gunes et al., 2009). Notch1 and DNMT3A are not only related to the proliferation of tumor cells, but also to the regulation of invasion and metastasis, which can influence patient prognosis (Gan et al., 2018; Xiao et al., 2020). It has been shown that patients with high 
COL2A1 expression have delayed recurrence (Ganapathi et al., 2016). Due to the lack of consistency in oncogene mutations in the two cohorts, further verification is needed. This inconsistency may be caused by an insufficient number of samples and differences in patient ethnicity.

Upon analysis of pathway enrichment, we found that a more favorable prognosis was related to the up-regulation of apoptosisrelated pathway, anti-tumor immune pathway, and EMT inhibition pathway (Figure 7). Activation of inositol-requiring enzyme $1 \alpha$ (IRE1 $\alpha$ ) can enhance platinum-mediated cell death and affect patients' drug sensitivity to platinum (Chen et al., 2019). P38 MAPK-Hsp27, which can be activated by platinumbased therapy, is another important pathway that regulates apoptosis and plays an important role in platinum-mediated cell death (Widmann et al., 1999; Chen et al., 2019). It has been shown that silencing the $\mathrm{P} 38$ protein results in a reduction of platinum-mediated apoptosis (Al-Khayal et al., 2020). Smad $2 / 3$ can be activated as result of the activation of TGF receptor by TGF- $\beta$, forming a complex with Smad4 (Derynck and Zhang, 2003). This is an important pathway affecting cell proliferation, differentiation and apoptosis, with the inhibition of Smad2 being shown to promote tumor metastasis (Ying et al., 2017). The expression of phosphorylated Smad2 (p-Smad2) is consistent with that of Smad4 in that a decrease in expression results in platinum resistance and poor prognosis (Huang et al., 2015; Liu et al., 2020; Wang et al., 2021). In both cases, this is due to the regulation of cell proliferation, adhesion and immune response. Therefore, the enrichment and up-regulation of the Smad2/3 nuclear pathway may improve patient prognosis and increase platinum drug sensitivity. Crosstalk mechanisms exist between the MAPK pathway and Smad pathways. TGF- $\beta$ can activate P38 MAPK pathway, and the activated MAPK can then activate Smad through direct phosphorylation or effector molecules, ultimately leading to an increase in platinum drug sensitivity (Javelaud and Mauviel, 2005). Rho protein family is an important group of molecules that regulate cell morphology, movement, adhesion and proliferation, as well as the actin cytoskeleton (Steichen et al., 2021). The activation of RhoA can reduce the mobility of post-EMT cells, while the inhibition of RhoA can lead to a shortened PFS and increase in lymphatic metastasis (Bellovin et al., 2005). Many other actin cytoskeleton regulators have also been found to inhibit EMT, such as lovastatin (Zheng et al., 2021), Rho GTPase activating protein 10 (ARHGAP10) (Lin et al., 2021) and Ras-related C3 botulinum toxin substrate 1B (RAC1B) (Ungefroren et al., 2020). Therefore, up-regulation of the actin cytoskeleton/RhoA regulation pathway may increase platinum drug sensitivity and improve prognosis by inhibiting EMT, reducing metastasis and regulating the number of cancer stem cells (Li et al., 2019). B cells also play an important role in anti-tumor immunity and are related to better prognosis (Tong et al., 2016; Cabrita et al., 2020; Helmink et al., 2020; Petitprez et al., 2020; Shen et al., 2020). Chemokines, such as CXCL-13 and CXCL-5, can cause B cells to aggregate at the tumor site (Hussain et al., 2021). The enhancement of chemokines and B cell immune activity may be another reason for the improved prognosis in the CAMSAP1-MT group.
Based on the analysis of cMAP datasets, anisomycin, econazole, etoposide, glimepiride and imatinib are the potential therapeutic drugs for SCLC patients with CAMSAP1 mutation. Anisomycin can inhibit angiogenesis, proliferation and invasion of tumor cells by blocking the PI3K/ Akt pathway (Ushijima et al., 2021)and Notch1 pathway (Ye et al., 2019). Econazole induces cell apoptosis and inhibits cancer invasion through the elevated protein level of p53 (Choi et al., 2020). Imatinib reduces the phosphorylation of the PDGFRa/Akt axis, suppressing tumor cell growth and migration (Nayeem et al., 2021). Etoposide, as a Topoisomerase inhibitor, has been approved by FDA and is widely used in cancer therapy (Montecucco et al., 2015). Glimepiride inhibits tumor cell growth through activation of AMPK(Long et al., 2020).

It should be noted that this study has some limitations. First of all, we did not compare the differences in the predictive effect of CAMSAP1 on platinum between different ethnicities. Secondly, the mechanism of how CAMSAP1 mutation affects drug sensitivity lacks experimental verification. Thirdly, the clinical characters of the two cohorts are not completely same and the number of samples in this study is insufficient, so the results should be verified in a larger population. Fourthly, the relationship between CAMAP1 mutation and other treatments could be further verified.

\section{CONCLUSION}

In this study, we showed that CAMSAP1 mutation can serve as a suitable biomarker of platinum drug sensitivity. We then went on to explore the possible mechanisms and found that CAMSAP1 mutation improves the sensitivity of platinum drugs and the prognosis of SCLC patients by regulating a variety of cellular activities. These include tumor cell growth, apoptosis, invasion, metastasis, anti-tumor immunity, and EMT. Our results suggest that gene mutation may be the molecular basis for the change in chemosensitivity. In addition, this study also provides important evidence for the guidance of treatment and clinical experimental design in SCLC and other types of tumors associated with CAMSAP1 mutation.

\section{DATA AVAILABILITY STATEMENT}

The datasets presented in this study can be found in online repositories. The names of the repository/repositories and accession number(s) can be found in the article/ Supplementary Material.

\section{AUTHOR CONTRIBUTIONS}

Writing-original draft, YY and AL; Conceptualization, PL and JZ; Investigation, AL; Writing-review and editing, YY, ZQ, AL, PL, QC, YW, and JZ; Formal analysis, YY and ZY; Visualization, YY and ZY. Correction, YY, ZY, AL, RS, YQ, 
TW, WS. All authors have read and agreed to the published version of the manuscript.

\section{FUNDING}

This work was supported by the Natural Science Foundation of Guangdong Province (Grant No. 2018A030313846 and 2021A1515012593), the Science and Technology Planning Project of Guangdong Province (Grant No. 2019A030317020) and the National Natural Science Foundation of China (Grant No. 81803067, 81802257, 81871859, 81772457, 82172750, and 82172811).

\section{REFERENCES}

Ahmed, N., Escalona, R., Leung, D., Chan, E., and Kannourakis, G. (2018). Tumour Microenvironment and Metabolic Plasticity in Cancer and Cancer Stem Cells: Perspectives on Metabolic and Immune Regulatory Signatures in Chemoresistant Ovarian Cancer Stem Cells. Semin. Cancer Biol. 53, 265-281. doi:10.1016/j.semcancer.2018.10.002

Al-Khayal, K., Vaali-Mohammed, M.-A., Elwatidy, M., Traiki, T. B., Al-Obeed, O., Azam, M., et al. (2020). Correction to: A Novel Coordination Complex of Platinum (PT) Induces Cell Death in Colorectal Cancer by Altering Redox Balance and Modulating MAPK Pathway. BMC Cancer 20 (1), 834. doi:10.1186/s12885-020-07245-x

Ardizzoni, A., Hansen, H., Dombernowsky, P., Gamucci, T., Kaplan, S., Postmus, P., et al. (1997). Topotecan, a New Active Drug in the Second-Line Treatment of Small-Cell Lung Cancer: A Phase II Study in Patients with Refractory and Sensitive Disease. The European Organization for Research and Treatment of Cancer Early Clinical Studies Group and New Drug Development Office, and the Lung Cancer Cooperative Group. J. Clin. Oncol. 15 (5), 2090-2096. doi:10.1200/JCO.1997.15.5.2090

Baines, A. J., Bignone, P. A., King, M. D. A., Maggs, A. M., Bennett, P. M., Pinder, J. C., et al. (2009). The CKK Domain (DUF1781) Binds Microtubules and Defines the CAMSAP/ssp4 Family of Animal Proteins. Mol. Biol. Evol. 26 (9), 2005-2014. doi:10.1093/molbev/msp115

Bellovin, D. I., Bates, R. C., Muzikansky, A., Rimm, D. L., and Mercurio, A. M. (2005). Altered Localization of P120 Catenin during Epithelial to Mesenchymal Transition of colon Carcinoma Is Prognostic for Aggressive Disease. Cancer Res. 65 (23), 10938-10945. doi:10.1158/ 0008-5472.CAN-05-1947

Cabrita, R., Lauss, M., Sanna, A., Donia, M., Skaarup Larsen, M., Mitra, S., et al. (2020). Tertiary Lymphoid Structures Improve Immunotherapy and Survival in Melanoma. Nature 577 (7791), 561-565. doi:10.1038/s41586019-1914-8

Chen, L., Jiang, K., Chen, H., Tang, Y., Zhou, X., Tan, Y., et al. (2019). Deguelin Induces Apoptosis in Colorectal Cancer Cells by Activating the P38 MAPK Pathway. Cancer Manag. Res. 11, 95-105. doi:10.2147/ CMAR.S169476

Choi, E. K., Park, E. J., Phan, T. T., Kim, H. D., Hoe, K.-L., and Kim, D.-U. (2020). Econazole Induces P53-dependent Apoptosis and Decreases Metastasis Ability in Gastric Cancer Cells. Biomolecules Ther. 28 (4), 370-379. doi:10.4062/ biomolther.2019.201

Chuang, M., Goncharov, A., Wang, S., Oegema, K., Jin, Y., and Chisholm, A. D. (2014). The Microtubule Minus-End-Binding Protein Patronin/PTRN-1 is Required for Axon Regeneration in C. Elegans. Cell Rep. 9 (3), 874-883. doi:10.1016/j.celrep.2014.09.054

Cui, L., Zhou, F., Chen, C., and Wang, C. C. (2019). Overexpression of CCDC69 Activates p14ARF/MDM2/p53 Pathway and Confers Cisplatin Sensitivity. J. Ovarian Res. 12 (1), 4. doi:10.1186/s13048-019-0479-3

David-Watine, B. (2011). Silencing Nuclear Pore Protein Tpr Elicits a Senescentlike Phenotype in Cancer Cells. PLoS One 6 (7), e22423. doi:10.1371/ journal.pone.0022423

\section{ACKNOWLEDGMENTS}

Special thanks to the English language polishing contributions from TopScience Editing.

\section{SUPPLEMENTARY MATERIAL}

The Supplementary Material for this article can be found online at: https://www.frontiersin.org/articles/10.3389/fcell.2021.770811/ full\#supplementary-material

Derynck, R., and Zhang, Y. E. (2003). Smad-dependent and Smad-independent Pathways in TGF- $\beta$ Family Signalling. Nature 425 (6958), 577-584. doi: $10.1038 /$ nature 02006

Farago, A. F., and Keane, F. K. (2018). Current Standards for Clinical Management of Small Cell Lung Cancer. Transl. Lung Cancer Res. 7 (1), 69-79. doi:10.21037/ tlcr.2018.01.16

Gan, R.-H., Wei, H., Xie, J., Zheng, D.-P., Luo, E.-L., Huang, X.-Y., et al. (2018). Notch1 Regulates Tongue Cancer Cells Proliferation, Apoptosis and Invasion. Cell Cycle 17 (2), 216-224. doi:10.1080/15384101.2017.1395534

Ganapathi, M. K., Jones, W. D., Sehouli, J., Michener, C. M., Braicu, I. E., Norris, E. J., et al. (2016). Expression Profile of COL2A1 and the Pseudogene SLC6A10P Predicts Tumor Recurrence in High-Grade Serous Ovarian Cancer. Int. J. Cancer 138 (3), 679-688. doi:10.1002/ijc.29815

George, J., Lim, J. S., Jang, S. J., Cun, Y., Ozretić, L., Kong, G., et al. (2015). Comprehensive Genomic Profiles of Small Cell Lung Cancer. Nature 524 (7563), 47-53. doi:10.1038/nature14664

Gong, T., Yan, Y., Zhang, J., Liu, S., Liu, H., Gao, J., et al. (2018). PTRN -1/ CAMSAP Promotes CYK -1/formin-dependent Actin Polymerization during Endocytic Recycling. EMBO J. 37 (9), e98556. doi:10.15252/embj.201798556

Govindan, R., Page, N., Morgensztern, D., Read, W., Tierney, R., Vlahiotis, A., et al. (2006). Changing Epidemiology of Small-Cell Lung Cancer in the United States over the Last 30 years: Analysis of the Surveillance, Epidemiologic, and End Results Database. J. Clin. Oncol. 24 (28), 4539-4544. doi:10.1200/ JCO.2005.04.4859

Hao, T., Wang, Z., Yang, J., Zhang, Y., Shang, Y., and Sun, J. (2020). MALAT1 Knockdown Inhibits Prostate Cancer Progression by Regulating miR-140/ BIRC6 axis. Biomed. Pharmacother. 123, 109666. doi:10.1016/ j.biopha.2019.109666

Helmink, B. A., Reddy, S. M., Gao, J., Zhang, S., Basar, R., Thakur, R., et al. (2020). B Cells and Tertiary Lymphoid Structures Promote Immunotherapy Response. Nature 577 (7791), 549-555. doi:10.1038/s41586-019-1922-8

Hendershott, M. C., and Vale, R. D. (2014). Regulation of Microtubule Minus-End Dynamics by CAMSAPs and Patronin. Proc. Natl. Acad. Sci. 111 (16), 5860-5865. doi:10.1073/pnas.1404133111

Hermann, P. C., Huber, S. L., Herrler, T., Aicher, A., Ellwart, J. W., Guba, M., et al. (2007). Distinct Populations of Cancer Stem Cells Determine Tumor Growth and Metastatic Activity in Human Pancreatic Cancer. Cell Stem Cell 1 (3), 313-323. doi:10.1016/j.stem.2007.06.002

Huang, C., Huang, M., Chen, W., Zhu, W., Meng, H., Guo, L., et al. (2015). $\mathrm{N}$-acetylglucosaminyltransferase V Modulates Radiosensitivity and Migration of Small Cell Lung Cancer through Epithelial-Mesenchymal Transition. FEBS J. 282 (22), 4295-4306. doi:10.1111/febs.13419

Hussain, M., Liu, J., Wang, G.-Z., and Zhou, G.-B. (2021). CXCL13 Signaling in the Tumor Microenvironment. Adv. Exp. Med. Biol. 1302, 71-90. doi:10.1007/9783-030-62658-7_6

Javelaud, D., and Mauviel, A. (2005). Crosstalk Mechanisms between the MitogenActivated Protein Kinase Pathways and Smad Signaling Downstream of TGF- $\beta$ : Implications for Carcinogenesis. Oncogene 24 (37), 5742-5750. doi:10.1038/ sj.onc. 1208928

Jett, J. R., Schild, S. E., Kesler, K. A., and Kalemkerian, G. P. (2013). Treatment of Small Cell Lung Cancer. Chest 143 (5 Suppl. 1), e400S-e419S. doi:10.1378/ chest.12-2363 
Kanzaki, H., Ouchida, M., Hanafusa, H., Yano, M., Suzuki, H., Aoe, M., et al. (2006). Single Nucleotide Polymorphism of the AXIN2 Gene Is Preferentially Associated with Human Lung Cancer Risk in a Japanese Population. Int. J. Mol. Med. 18 (2), 279-284. doi:10.3892/ijmm.18.2.279

Li, M., Lin, A., Luo, P., Shen, W., Xiao, D., Gou, L., et al. (2020). DNAH10 Mutation Correlates with Cisplatin Sensitivity and Tumor Mutation burden in Small-Cell Lung Cancer. Aging 12 (2), 1285-1303. doi:10.18632/aging.102683

Li, N., Babaei-Jadidi, R., Lorenzi, F., Spencer-Dene, B., Clarke, P., Domingo, E., et al. (2019). An FBXW7-ZEB2 axis Links EMT and Tumour Microenvironment to Promote Colorectal Cancer Stem Cells and Chemoresistance. Oncogenesis 8 (3), 13. doi:10.1038/s41389-019-0125-3

Li, Q., Damish, A. W., Frazier, Z., Liu, D., Reznichenko, E., Kamburov, A., et al. (2019). ERCC2 Helicase Domain Mutations Confer Nucleotide Excision Repair Deficiency and Drive Cisplatin Sensitivity in Muscle-Invasive Bladder Cancer. Clin. Cancer Res. 25 (3), 977-988. doi:10.1158/10780432.CCR-18-1001

Lin, H., Guo, Q., Lu, S., Chen, J., Li, X., Gong, M., et al. (2019). LncRNA SUMO1P3 Promotes Proliferation and Inhibits Apoptosis in Colorectal Cancer by Epigenetically Silencing CPEB3. Biochem. Biophysical Res. Commun. 511 (2), 239-245. doi:10.1016/j.bbrc.2019.02.006

Liu, N., Qi, D., Jiang, J., Zhang, J., and Yu, C. (2020). Expression Pattern of $\mathrm{p}$-Smad2/Smad4 as a P-redictor of S-urvival in I-nvasive B-reast D-uctal C-arcinoma. Oncol. Lett. 19 (3), 1789-1798. doi:10.3892/ol.2020.11297

Lohse, I., Borgida, A., Cao, P., Cheung, M., Pintilie, M., Bianco, T., et al. (2015). BRCA1 and BRCA2 Mutations Sensitize to Chemotherapy in Patient-Derived Pancreatic Cancer Xenografts. Br. J. Cancer 113 (3), 425-432. doi:10.1038/ bjc.2015.220

Long, L., Hu, X., Li, X., Zhou, D., Shi, Y., Wang, L., et al. (2020). The Anti-breast Cancer Effect and Mechanism of Glimepiride-Metformin Adduct. Onco Targets Ther. 13, 3777-3788. doi:10.2147/OTT.S240252

Luo, Y., Tong, L., Meng, H., Zhu, W., Guo, L., Wei, T., et al. (2017). MiR-335 Regulates the Chemo-Radioresistance of Small Cell Lung Cancer Cells by Targeting PARP-1. Gene 600, 9-15. doi:10.1016/j.gene.2016.11.031

Ma, C., Ma, C., Chen, H. I., Flores, M., Huang, Y., Chen, Y., et al. (2013). BRCAMonet: A Breast Cancer Specific Drug Treatment Mode-Of Action Network for Treatment Effective Prediction Using Large Scale Microarray Database. BMC Syst. Biol. 7 (Suppl 5), S5. doi:10.1186/1752-0509-7-S5-S5

Maji, S., Panda, S., Samal, S. K., Shriwas, O., Rath, R., Pellecchia, M., et al. (2018). Bcl-2 Antiapoptotic Family Proteins and Chemoresistance in Cancer. Adv. Cancer Res. 137, 37-75. doi:10.1016/bs.acr.2017.11.001

Mani, J., Vallo, S., Rakel, S., Antonietti, P., Gessler, F., Blaheta, R., et al. (2015). Chemoresistance Is Associated with Increased Cytoprotective Autophagy and Diminished Apoptosis in Bladder Cancer Cells Treated with the BH3 Mimetic (-)-Gossypol (AT-101). BMC Cancer 15, 224. doi:10.1186/s12885-015-1239-4

Montecucco, A., Zanetta, F., and Biamonti, G. (2015). Molecular Mechanisms of Etoposide. EXCLI J. 14, 95-108. doi:10.17179/excli2015-561

Nayeem, M. J., Yamamura, A., Hayashi, H., Muramatsu, H., Nakamura, K., Sassa, N., et al. (2022). Imatinib Mesylate Inhibits Androgen-independent PC-3 Cell Viability, Proliferation, Migration, and Tumor Growth by Targeting PlateletDerived Growth Factor Receptor- $\alpha$. Life Sci. 288, 120171. doi:10.1016/ j.lfs.2021.120171

Ogino, H., Hanibuchi, M., Kakiuchi, S., Saijo, A., Tezuka, T., Toyoda, Y., et al. (2016). Analysis of the Prognostic Factors of Extensive Disease Small-Cell Lung Cancer Patients in Tokushima university Hospital. J. Med. Invest. 63 (3-4), 286-293. doi:10.2152/jmi.63.286

Petitprez, F., de Reyniès, A., Keung, E. Z., Chen, T. W.-W., Sun, C.-M., Calderaro, J., et al. (2020). B Cells Are Associated with Survival and Immunotherapy Response in Sarcoma. Nature 577 (7791), 556-560. doi:10.1038/s41586-0191906-8

Pinarbasi, E. G., Pinarbasi, E., Pinarbasi, H., and Silig, Y. (2009). Strong Association between Lung Cancer and the AXIN2 Polymorphism. Mol. Med. Rep. 02 (6), 1029-1035. doi:10.3892/mmr_00000210

Qiu, Z., Lin, A., Li, K., Lin, W., Wang, Q., Wei, T., et al. (2019a). A Novel Mutation Panel for Predicting Etoposide Resistance in Small-Cell Lung Cancer. Drug Des. Devel Ther. 13, 2021-2041. doi:10.2147/DDDT.S205633
Qiu, Z., Zhu, W., Meng, H., Tong, L., Li, X., Luo, P., et al. (2019b). CDYL Promotes the Chemoresistance of Small Cell Lung Cancer by Regulating H3K27 Trimethylation at the CDKN1C Promoter. Theranostics 9 (16), 4717-4729. doi: $10.7150 /$ thno. 33680

Rossi, A., Di Maio, M., Chiodini, P., Rudd, R. M., Okamoto, H., Skarlos, D. V., et al. (2012). Carboplatin- or Cisplatin-Based Chemotherapy in First-Line Treatment of Small-Cell Lung Cancer: The COCIS Meta-Analysis of Individual Patient Data. J. Clin. Oncol. 30 (14), 1692-1698. doi:10.1200/JCO.2011.40.4905

Sabari, J. K., Lok, B. H., Laird, J. H., Poirier, J. T., and Rudin, C. M. (2017). Unravelling the Biology of SCLC: Implications for Therapy. Nat. Rev. Clin. Oncol. 14 (9), 549-561. doi:10.1038/nrclinonc.2017.71

Shen, W., Zhang, W., Ye, W., Wang, H., Zhang, Q., Shen, J., et al. (2020). SR9009 Induces a REV-ERB Dependent Anti-small-cell Lung Cancer Effect through Inhibition of Autophagy. Theranostics 10 (10), 4466-4480. doi:10.7150/thno.42478

Steichen, C., Hervé, J. C., Hauet, T., and Bourmeyster, N. (2021). Rho GTPases in Kidney Physiology and Diseases. Small GTPases 17, 1-21. doi:10.1080/ 21541248.2021.1932402

Tong, L., Luo, Y., Wei, T., Guo, L., Wang, H., Zhu, W., et al. (2016). KH-type Splicing Regulatory Protein (KHSRP) Contributes to Tumorigenesis by Promoting miR-26a Maturation in Small Cell Lung Cancer. Mol. Cel. Biochem. 422 (1-2), 61-74. doi:10.1007/s11010-016-2806-y

Tsoukalas, N., Aravantinou-Fatorou, E., Baxevanos, P., Tolia, M., Tsapakidis, K., Galanopoulos, M., et al. (2018). Advanced Small Cell Lung Cancer (SCLC): New Challenges and New Expectations. Ann. Transl. Med. 6 (8), 145. doi:10.21037/atm.2018.03.31

Ungefroren, H., Wellner, U. F., Keck, T., Lehnert, H., and Marquardt, J.-U. (2020). The Small GTPase RAC1B: A Potent Negative Regulator Of-And Useful Tool to Study-Tgf $\beta$ Signaling. Cancers 12 (11), 3475. doi:10.3390/ cancers 12113475

Ushijima, H., Monzaki, R., and Funakoshi, M. (2021). Analysis of Differentially Expressed Genes Responsible for the Suppressive Effect of Anisomycin on Cell Proliferation of DLD-1 Cells. Biochem. Biophys. Rep. 27, 101038. doi:10.1016/ j.bbrep.2021.101038

Wang, J., Mi, J.-Q., Debernardi, A., Vitte, A.-L., Emadali, A., Meyer, J. A., et al. (2015). A Six Gene Expression Signature Defines Aggressive Subtypes and Predicts Outcome in Childhood and Adult Acute Lymphoblastic Leukemia. Oncotarget 6 (18), 16527-16542. doi:10.18632/oncotarget.4113

Wang, Q., Zeng, F., Sun, Y., Qiu, Q., Zhang, J., Huang, W., et al. (2018). Etk Interaction with PFKFB4 Modulates Chemoresistance of Small-Cell Lung Cancer by Regulating Autophagy. Clin. Cancer Res. 24 (4), 950-962. doi:10.1158/1078-0432.CCR-17-1475

Wang, Y., Xue, Q., Zheng, Q., Jin, Y., Shen, X., Yang, M., et al. (2021). SMAD4 Mutation Correlates with Poor Prognosis in Non-small Cell Lung Cancer. Lab. Invest. 101 (4), 463-476. doi:10.1038/s41374-020-00517-x

Waqar, S. N., and Morgensztern, D. (2017). Treatment Advances in Small Cell Lung Cancer (SCLC). Pharmacol. Ther. 180, 16-23. doi:10.1016/ j.pharmthera.2017.06.002

Widmann, C., Gibson, S., Jarpe, M. B., and Johnson, G. L. (1999). Mitogenactivated Protein Kinase: Conservation of a Three-Kinase Module from Yeast to Human. Physiol. Rev. 79 (1), 143-180. doi:10.1152/ physrev.1999.79.1.143

Xiao, J., Liu, Y., Wu, F., Liu, R., Xie, Y., Yang, Q., et al. (2020). RETRACTED: miR639 Expression Is Silenced by DNMT3A-Mediated Hypermethylation and Functions as a Tumor Suppressor in Liver Cancer Cells. Mol. Ther. 28 (2), 587-598. doi:10.1016/j.ymthe.2019.11.021

Xu, S., Tao, Z., Hai, B., Liang, H., Shi, Y., Wang, T., et al. (2016). MiR-424(322) Reverses Chemoresistance via T-Cell Immune Response Activation by Blocking the PD-L1 Immune Checkpoint. Nat. Commun. 7, 11406. doi:10.1038/ ncomms 11406

Yau, K. W., van Beuningen, S. F. B., Cunha-Ferreira, I., Cloin, B. M. C., van Battum, E. Y., Will, L., et al. (2014). Microtubule Minus-End Binding Protein CAMSAP2 Controls Axon Specification and Dendrite Development. Neuron 82 (5), 1058-1073. doi:10.1016/ j.neuron.2014.04.019

Ye, W., Ni, Z., Yicheng, S., Pan, H., Huang, Y., Xiong, Y., et al. (2019). Anisomycin Inhibits Angiogenesis in Ovarian Cancer by Attenuating the Molecular Sponge 
Effect of the lncRNA-Meg3/miR-421/PDGFRA axis. Int. J. Oncol. 55 (6), 1296-1312. doi:10.3892/ijo.2019.4887

Ying, Z., Tian, H., Li, Y., Lian, R., Li, W., Wu, S., et al. (2017). CCT6A Suppresses SMAD2 and Promotes Prometastatic TGF- $\beta$ Signaling. J. Clin. Invest. 127 (5), 1725-1740. doi:10.1172/JCI90439

Yu, J., Liu, D., Sun, X., Yang, K., Yao, J., Cheng, C., et al. (2019). CDX2 Inhibits the Proliferation and Tumor Formation of colon Cancer Cells by Suppressing Wnt/ $\beta$-Catenin Signaling via Transactivation of GSK- $3 \beta$ and Axin2 Expression. Cell Death Dis 10 (1), 26. doi:10.1038/s41419-018-1263-9

Zhang, J., Zhou, N., Lin, A., Luo, P., Chen, X., Deng, H., et al. (2021). ZFHX3 Mutation as a Protective Biomarker for Immune Checkpoint Blockade in Nonsmall Cell Lung Cancer. Cancer Immunol. Immunother. 70 (1), 137-151. doi:10.1007/s00262-020-02668-8

Zheng, C., Yan, S., Lu, L., Yao, H., He, G., Chen, S., et al. (2021). Lovastatin Inhibits EMT and Metastasis of Triple-Negative Breast Cancer Stem Cells through Dysregulation of Cytoskeleton-Associated Proteins. Front. Oncol. 11, 656687. doi:10.3389/fonc.2021.656687
Conflict of Interest: The authors declare that the research was conducted in the absence of any commercial or financial relationships that could be construed as a potential conflict of interest.

Publisher's Note: All claims expressed in this article are solely those of the authors and do not necessarily represent those of their affiliated organizations, or those of the publisher, the editors and the reviewers. Any product that may be evaluated in this article, or claim that may be made by its manufacturer, is not guaranteed or endorsed by the publisher.

Copyright (c) 2022 Yi, Qiu, Yao, Lin, Qin, Sha, Wei, Wang, Cheng, Zhang, Luo and Shen. This is an open-access article distributed under the terms of the Creative Commons Attribution License (CC BY). The use, distribution or reproduction in other forums is permitted, provided the original author(s) and the copyright owner(s) are credited and that the original publication in this journal is cited, in accordance with accepted academic practice. No use, distribution or reproduction is permitted which does not comply with these terms. 